\title{
Density Profiles of Gases and Fluids in Gravitational Potentials from a Generalization of Hydrostatic Equilibrium
}

\author{
Richard B. Holmes \\ The Boeing Company, Chicago, USA \\ Email: rholmes001@aol.com
}

How to cite this paper: Holmes, R.B. (2020) Density Profiles of Gases and Fluids in Gravitational Potentials from a Generalization of Hydrostatic Equilibrium. Journal of Modern Physics, 11, 648-667. https://doi.org/10.4236/jmp.2020.115042

Received: March 13, 2020

Accepted: May 5, 2020

Published: May 8, 2020

Copyright $\odot 2020$ by author(s) and Scientific Research Publishing Inc. This work is licensed under the Creative Commons Attribution International License (CC BY 4.0).

http://creativecommons.org/licenses/by/4.0/

\section{(c) (i) Open Access}

\begin{abstract}
It is well-known that equilibrium density profiles of gases and fluids in gravitational potentials have an $r^{-1}$ dependence, where $r$ is the radius. This is found in both astronomical observations and detailed simulations in spherically-symmetric geometries. It is also well-known that the standard equation for hydrostatic equilibrium does not produce such solutions. This paper utilizes a Lagrangian formulation that produces a closed-form $r^{-1}$ solution and identifies the needed terms to be added to the standard equation for hydrostatic equilibrium to obtain such a solution. Variants of the $r^{-1}$ solution avoid a density singularity at the origin and a total-enclosed mass singularity at infinity. The resulting solutions are shown to be in good agreement with well-established density profiles of ordinary matter in galaxies, dark matter haloes, and the atmosphere of earth. Comparisons are made to solutions based on the standard hydrostatic equation, including solutions based on the Lane-Emden equation. The origins of differences are explained.
\end{abstract}

\section{Keywords}

Density Profiles, Gravitational Potentials, Dark Matter

\section{Introduction}

Early numerical solutions for dark matter (DM) density profiles were found to have dependence inversely proportional to radius [1]. Astronomical observations of both ordinary matter and dark matter have also inferred density profiles closely resembling the $r^{-1}$ profile, where $r$ is the radius from the nominal center of the distribution, in a region surrounding the origin [2] [3] [4] [5] [6]. However, the profile has several serious drawbacks. First, it gives infinite density at the 
origin. Second, it gives infinite total enclosed mass. Third, it is not consistent with many observations of the shape of the inner profiles of low-spectral brightness galaxies [7] [8] [9]. Nonetheless, the $r^{-1}$ profile is "ubiquitous" [10]. This paper will provide theoretical support that such a profile is indeed ubiquitous when there is thermal and mechanical equilibrium.

To analyze the spatial distribution of a gas of particles, N-body simulations [1] [11] or solutions to the Vlasov equation [12] are typically used. Two simplified governing equations are considered for DM density profiles in this paper. The first is the standard equation for hydrostatic equilibrium in a spherically-symmetric geometry:

$$
(1 / \rho)(\mathrm{d} P / \mathrm{d} r)=-m_{v} M_{\text {enc }}(r) G / r^{2} .
$$

here $\rho$ is the number density of DM, $P$ is the pressure, $r$ is the radius, $m_{v}$ is the mass of a DM particle, $M_{\text {enc }}(r)$ is the enclosed mass, and $G$ is the gravitational constant. This equation can be solved using the well-known Lane-Emden formulation [13], which uses the assumption that pressure is a function of density only:

$$
P=c_{\gamma} \rho^{\gamma},
$$

where $c_{\gamma}$ is a constant for a given polytropic exponent $\gamma$. An inhomogeneous form of the Lane-Emden equation can be used when ordinary matter (OM) is present as shown later in the paper. As shown in Section 4, calculations using Equations (1) and (2) are inconsistent with an $r^{-1}$ solution. Such solutions have no cusp at the origin and are a poor match to the standard Sersic or Einasto profiles based on observations [1] [2] [3] [4] [6] [14] [15] [16]. This is found to be true for any polytropic exponent between about 1.1 and 2 in Section 4 below.

To address this, a generalization of Equation (1) is derived using a Lagrangian formulation. This derivation is given in Section 2. A partial validation of this derivation is given in Section 3 using the properties of a well-known density profile of a gas in a gravitational potential, namely, the earth's atmosphere. Section 4 presents behavior of solutions near the origin. Section 5 presents the behavior of solutions far from the origin and presents a sample calculation for a dark-matter halo. Section 6 compares the solutions to Einasto and de-projected Sersic profiles. Section 7 compares the solutions to results from the Lane Emden equation. Section 8 provides a brief summary.

\section{Derivation of a Generalized Equation for Hydrostatic Equilibrium}

The assumed kinetic and potential energy densities for a medium in a spherically symmetric geometry under the influence of gravity is given by

$$
\text { K.E. }(r)=P(r) \text {, P.E. }(r)=-\rho(r) m_{v} M_{\text {enc }}(r) G / r \text {, }
$$

where the variables are defined as in Equation (1). In the topic of this paper, $M_{\text {enc }}(r)$ consists of both ordinary and dark matter. The ordinary (radiant) 
matter has mass density denoted $\rho_{m, \text { rad }}(r)$. Note that the pressure is treated as a kinetic energy for the obvious reason. This will allow a variational approach with a Lagrangian density. Explicit relativistic effects and the possible impact of angular momentum are neglected in this initial analysis

The pressure is assumed to be a function of density only. As an example, the number density for fermions in Equation (3) assuming thermodynamic equilibrium is

$$
\rho=n_{s} /\left(2 \pi^{2} \hbar^{3}\right) \int p^{2} \mathrm{~d} p /\left[\exp \left(\left\{\left[(p c)^{2}+\left(m_{v} c^{2}\right)^{2}\right]^{1 / 2}-m_{v} c^{2}-\mu_{v}\right\} / k T\right)+1\right]
$$

where $p$ is the fermion momentum, $m_{v}$ is its mass, $n_{s}$ is the number of spin states, $\mu_{v}$ is the chemical potential, and $\hbar$ is Planck's constant. The other variables have been defined earlier. In Equation (4), the chemical potential is set to zero for non-interacting particles. With Equation (4) the pressure for fermions has the usual form in the non-relativistic limit, and is given by

$$
P=c_{5 / 3} \rho^{5 / 3},
$$

where $c_{5 / 3}=1.914 \hbar^{2} / m_{v}$ assuming 2 spin states for a fermion. For the relativistic case, the exponent is $4 / 3$, and $c_{4 / 3}=5.536 \hbar c$. For the general case of arbitrary polytropic exponent $\gamma$ and assuming $c_{\gamma}$ is of the form $\alpha \hbar^{a} c^{b} m_{v}^{c}$ with $\alpha$ a dimensionless constant of order unity, one obtains $c_{\gamma}=\alpha \hbar^{3(\gamma-1)} c^{5-3 \gamma} m_{v}^{4-3 \gamma}$. With this relation and $m_{v}=1 \mathrm{eV} / c^{2}$, for example, one obtains $c_{5 / 3}=7.42 \times 10^{-32} \mathrm{~J} \cdot \mathrm{m}^{-2}$ For $\gamma=1$ and 2 one finds $c_{1} \approx m_{v} c^{2}=16 \times 10^{-20} \mathrm{~J}$ and $c_{2} \approx \hbar^{3} /\left(m_{v}^{2} c\right)=1.22 \times 10^{-39} \mathrm{~J} \cdot \mathrm{m}^{3}$, respectively.

This initial general development also considers independent variations of pressure with density and temperature, $(\partial P / \partial \rho)_{T}$ and $(\partial P / \partial T)_{\rho}$ Moving on to the second term of Equation (3), the gravitational potential energy term is of the usual form, involving the enclosed mass computed starting from the center of mass of the overall mass distribution. It also has units of energy density. One may use the kinetic and potential energy density expressions of Equation (3) to find the density of DM or OM versus radius in a spherically-symmetric geometry for a single species of matter. This is done by taking variations of the Lagrangian density, K.E.-P.E. with respect to $\rho$ and T. The result for DM or OM is (with ordinary matter or dark matter held fixed, respectively)

$$
\begin{aligned}
& {\left[(\partial P / \partial \rho)_{T}+m_{v} M_{e n c}(r) G / r\right] \delta \rho(r)+4 \pi \rho m_{v}^{2}\left[\int_{0}^{r} r^{\prime 2} \mathrm{~d} r^{\prime} \delta \rho\left(r^{\prime}\right)\right] G / r} \\
& +(\partial P / \partial T)_{\rho} \delta T(r)=0 .
\end{aligned}
$$

This equation should hold for arbitrary small variations $\delta \rho(r)$ and $\delta T(r)$ at each point $r$ in mechanical and thermodynamic equilibrium at temperature $T(r)$ and density $\rho(r)$. The two last terms look like they could pose a problem. To address the last term, assume Equation (2) applies, so that $T$ is a function of $\rho$ only, and $\delta T(r)=(\mathrm{d} T / \mathrm{d} \rho) \delta \rho(r)$. Then 


$$
\begin{aligned}
& {\left[(\partial P / \partial \rho)_{T}+(\partial P / \partial T)_{\rho} \mathrm{d} T / \mathrm{d} \rho+m_{v} M_{\text {enc }}(r) G / r\right] \delta \rho(r)} \\
& +4 \pi \rho m_{v}^{2}\left[\int_{0}^{r} r^{\prime 2} \mathrm{~d} r^{\prime} \delta \rho\left(r^{\prime}\right)\right] G / r .
\end{aligned}
$$

Note that the first two terms are $\mathrm{d} P / \mathrm{d} \rho$. To address the last term, differentiate Equation (7) with respect to $r$ to obtain

$$
\begin{aligned}
& {\left[\mathrm{d} / \mathrm{d} r(\partial P / \partial \rho)+4 \pi r^{2} m_{v}\left(\rho m_{v}+\rho_{m, r a d}\right) G / r-m_{v} M_{e n c}(r) G / r^{2}\right.} \\
& \left.+4 \pi \rho m_{v}^{2} r^{2} G / r\right] \delta \rho(r)+\left[\mathrm{d} P / \mathrm{d} \rho+m_{v} M_{e n c}(r) G / r\right] \mathrm{d} \delta \rho(r) / \mathrm{d} r \\
& +[\mathrm{d} / \mathrm{d} r(\rho / r)] m_{v}^{2} G\left[4 \pi \int_{0}^{r} r^{\prime 2} \mathrm{~d} r^{\prime} \delta \rho\left(r^{\prime}\right)\right] .
\end{aligned}
$$

The integral in the last term in Equation (8) can then be eliminated using Equation (7) to give

$$
\begin{aligned}
& {\left[\mathrm{d} / \mathrm{d} r(\partial P / \partial \rho)+4 \pi r^{2} m_{v}\left(2 \rho m_{v}+\rho_{m, r a d}\right) G / r-m_{v} M_{e n c}(r) G / r^{2}\right] \delta \rho(r)} \\
& \left.+4 \pi \rho m_{v}^{2} r^{2} G / r\right] \delta \rho(r)+\left[\mathrm{d} P / \mathrm{d} \rho+m_{v} M_{\text {enc }}(r) G / r\right] \mathrm{d} / \mathrm{d} r(\delta \rho(r)) \\
& -(\rho / r)^{-1}[\mathrm{~d} / \mathrm{d} r(\rho / r)]\left[\mathrm{d} P / \mathrm{d} \rho+m_{v} M_{e n c}(r) G / r\right] \delta \rho(r)=0 .
\end{aligned}
$$

The second term in Equation (9) is the change in the potential energy of the system at radius $r$ due to mass at radius $r$, and can be dropped if the enclosed mass is understood to be the enclosed mass strictly less than $r$.

To address the term involving $\mathrm{d} / \mathrm{d} r[\delta \rho(r)]$ the second line in Equation (9) can be integrated by parts, accounting for the volume integral of the Lagrangian density, as well as the $r^{2}$ factor that is associated with it. After considerable algebra, the result is

$$
\begin{aligned}
& \left\{\left[\mathrm{d} P / \mathrm{d} \rho+m_{v} M_{e n c}(r) G / r\right](1 / \rho) \mathrm{d} \rho / \mathrm{d} r\right. \\
& \left.+\left[m_{v} M_{e n c}(r) G / r+\mathrm{d} P / \mathrm{d} \rho\right] / r\right\} \delta \rho(r)=0 .
\end{aligned}
$$

This equation now has the required form so that the expression in curly brackets is zero:

$$
\begin{aligned}
& (1 / \rho) \mathrm{d} P / \mathrm{d} r+(1 / \rho)(\mathrm{d} \rho / \mathrm{d} r)\left[m_{v} M_{e n c}(r) G / r\right] \\
& =-m_{v} M_{\text {enc }}(r) G / r^{2}-(\mathrm{d} P / \mathrm{d} \rho) / r .
\end{aligned}
$$

Equation (11) is the result for strict equilibrium for a medium with a pressure that depends on density only in a spherically-symmetric geometry, and with a single species of matter. The limiting case of the standard hydrostatic equation is obtained by neglect of the second terms on both sides of Equation (11).

Based on the usual derivation of Equation (1), the standard hydrostatic equation should be valid when mechanical equilibrium applies but the stricter action equilibrium of Equation (11) does not apply. The term involving $(1 / \rho)(\mathrm{d} \rho / \mathrm{d} r)$ in Equation (11) arises because of the energy required to maintain a density gradient in a gravity potential, and the term involving $-(\mathrm{d} P / \mathrm{d} \rho) / r$ arises because of the form of the divergence of the radial part of the bulk modulus in a spherical geometry. 
It is also worth noting that Equation (11) can be written as

$$
\mathrm{d} \ln (\rho) / \mathrm{d} \ln (r)=-1 \text {. }
$$

This simplification indicates a solution for density of the form

$$
\rho \sim \rho_{0}\left(r_{0} / r\right) \text {. }
$$

This result is both remarkable and perhaps expected. It arises because of the spherical geometry and the $r^{-1}$ potential as discussed above. It matches the $\mathrm{Na}$ varro-Frenk-White (NFW) result [1] as well as other related results near the origin [2] [3] [5]. However, it has two serious drawbacks. First, it gives infinite density at the origin. Second, it gives infinite total enclosed mass. One may attempt to address these issues within the Lagrangian formalism by introducing a density constraint using a Lagrange multiplier $\lambda_{\rho}$, and a total particle number (mass) constraint with multiplier $\lambda_{M}$. With these two constraints, Equation (10) becomes

$$
\begin{aligned}
& \left\{\left[1+r \lambda_{M} /\left(\rho m_{v}^{2} G\right)\right]^{-1}[(1 / \rho) \mathrm{d} \rho / \mathrm{d} r]\left[\mathrm{d} P / \mathrm{d} \rho+m_{v} M_{\text {enc }}(r) G / r+\lambda_{\rho}\right]\right\} \delta \rho(r) \\
& +\left(\left\{2-\left[1+r \lambda_{M} / \rho m_{v}^{2} G\right]^{-1}\right\}\left[\mathrm{d} P / \mathrm{d} \rho+m_{v} M_{e n c}(r) G / r+\lambda_{\rho}\right] / r\right) \delta \rho(r) \\
& +\left[\lambda_{M}\left(4 \pi m_{v}^{2} G\right) \mathrm{d} / \mathrm{d} r(\rho / r)\right] \int_{r}^{\infty} \mathrm{d} r^{\prime} r^{\prime 2} \delta \rho\left(r^{\prime}\right) /\left[\lambda_{M}+\rho m_{v}^{2} G / r\right]=0 .
\end{aligned}
$$

The last term arises from the variation of the total-mass constraint with respect to density. For $r$ small (close to the origin) or large (far from the origin), one might expect this integral to be approximately zero, since the total mass constraint requires that the radial integral from zero to infinity of the density variations be identically zero. This version of the equation will be explored in subsequent sections of this paper.

\section{Generalized Equation for Earth's Atmospheric Density Profile with Altitude}

As a basic check of the results of Section 2, one may consider the case of earth's atmosphere. In the earth's atmosphere, it is well-known that (a) the temperature is not constant with altitude in the troposphere [17], and (b), that despite (a), a density profile computed with an isothermal atmosphere is a decent approximation. This apparent inconsistency is investigated in this section with various solutions and in the process provides some confirmation that the generalized hydrostatic equation has validity.

First the version of the generalized equation for hydrostatic equilibrium given by Equation (11) is considered. For the earth's atmosphere at distance $z$ above sea level, one has

$$
(1 / \rho) \mathrm{d} P / \mathrm{d} r+(1 / \rho)(\mathrm{d} \rho / \mathrm{d} z) m_{\text {air }} g z=-m_{\text {air }} g-\mathrm{d} P / \mathrm{d} \rho /\left(r_{e}+z\right) .
$$

here $m_{\text {air }}$ is the atomic weight of air, approximately $(29.0)\left(1.67 \times 10^{-27}\right) \mathrm{kg}$, and $g$ is the gravitational constant at or near earth's surface, $9.8 \mathrm{~m} \cdot \mathrm{sec}^{-2}$. The radius $r_{e}$ is measured from earth center. Next consider an equation of state 


$$
P(z)=k \rho(z) T(z),
$$

where $T(z)$ is the temperature, which is treated as an input from [17] for some of the following equations. Note that $(\mathrm{d} P / \mathrm{d} \rho) / r_{e}=k T(\mathrm{z}) / r_{e}$ is about $6.3 \times 10^{-28}$ $\mathrm{J} \cdot \mathrm{m}^{-1}$ and that $m_{\text {air }} g=4.7 \times 10^{-25} \mathrm{~J} \cdot \mathrm{m}^{-1}$. Hence the last term of Equation (15) can be neglected relative to $m_{\text {air }} g$. Using expression (16) and Equation (15) one obtains

$$
(k / \rho)[\mathrm{d} \rho / \mathrm{d} z T(z)+\rho(z) \mathrm{d} T / \mathrm{d} z]+(1 / \rho)(\mathrm{d} \rho / \mathrm{d} z) m_{\text {air }} g z=-m_{\text {air }} g .
$$

This equation simplifies to (neglecting terms of order $z / r_{e}$ ),

$$
(1 / \rho)(\mathrm{d} \rho / \mathrm{d} z)=\left[-m_{\text {air }} g-k \mathrm{~d} T / \mathrm{d} z\right] /\left[k T(z)+m_{\text {air }} g z\right] .
$$

This can be solved analytically:

$$
\rho(z)=\rho_{0} k T(z=0) /\left[k T(z)+m_{\text {air }} g z\right] .
$$

here $\rho_{0}$ is the density at sea level, which is an input. The solution of Equation (19) gives poor agreement with measured data, with significantly larger density than measured at higher altitudes. This will be seen to arise from the underlying assumption of strict action (mechanical and thermal) equilibrium. Equation (19) may be compared to the isothermal approximation, which amounts to retention of only the first terms on both sides of Equation (17) and assuming constant temperature. One obtains the usual exponential atmosphere in this case,

$$
\rho(z)=\rho_{0} \exp \left[-m_{\text {air }} g z / k T_{0}\right],
$$

where $T_{0}$ is the temperature of the atmosphere at sea level.

There is also the adiabatic equation for pressure and density. The above Equations (17)-(19) assumes strict action equilibrium. The adiabatic equation assumes that hot parcels of air near the surface of the earth rise without significant heat exchange with the surrounding air. Hence it corresponds to a process in quasi-static equilibrium that is not in strict mechanical and thermal equilibrium at all layers. With this insight, the adiabatic result can be derived from Equation (17) by neglect of the last terms on both sides of the equation, and assuming the gas satisfies a polytropic relation (2) with a polytropic exponent $\gamma \sim 1.4$. With these assumptions, one obtains the result from the standard hydrostatic equation, which is the well-known formula for density [18]:

$$
\rho(z)=\rho_{0}\left[1-m_{\text {air }} g z /\left(c_{p} T_{0}\right)\right]^{1 /(\gamma-1)} .
$$

here $c_{p}$ is the specific heat at constant pressure of the atmosphere at sea level. Note that $c_{p} T_{0}=[(\gamma-1) / \gamma] P_{0} / \rho_{0}$, where $P_{0}$ is the pressure at sea level. For Figure 1 below, the value of $c_{p}$ used is $1004.7 \mathrm{~J} \cdot \mathrm{kg}^{-1} \cdot \mathrm{K}^{-1}$, the value of $T_{0}$ used is 290.1 $\mathrm{K}$. The latter is consistent with the ideal gas law and the sea-level values of pressure and density given in [17]. For Figure 1, a polytropic exponent of 1.38 rather than 1.40 is used and is justifiable because of the presence of significant water vapor in the middle atmosphere between 1 and $15 \mathrm{~km}$. The integration step size is $250 \mathrm{~m}$. 

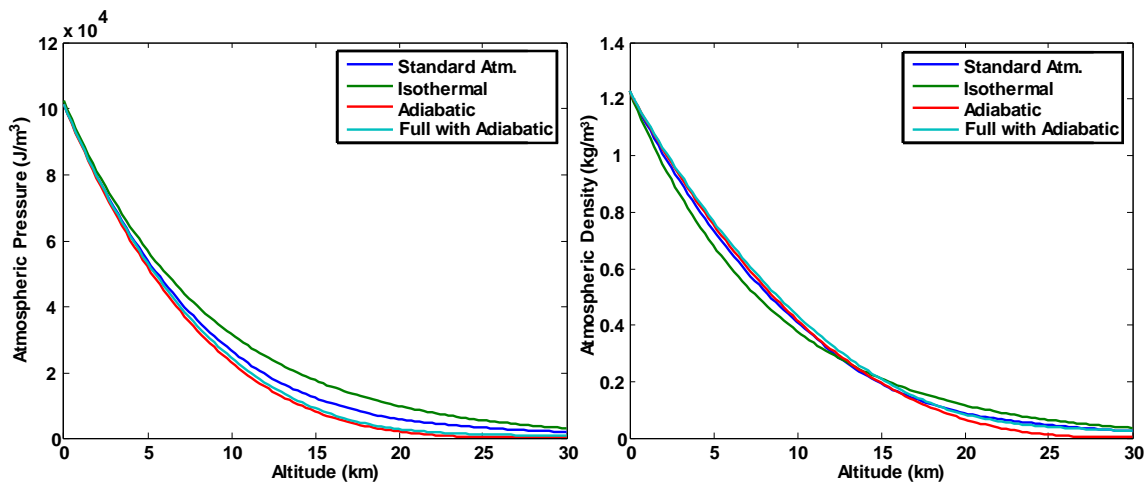

Figure 1. Solutions for Earth's atmospheric pressure (a) and density (b) versus altitude under various assumptions. Blue: standard atmospheric density (measured). Green: isothermal profile from Equation (20) with $T=290.1 \mathrm{~K}$. Red: Adiabatic result (21) using the standard hydrostatic equation. Cyan: Adiabatic result using generalized hydrostatic Equation (15) with factor given by Equation (22).

It should be noted that Equation (21) gives nonsensical results for $m_{\text {air }} g z /\left(c_{p} T_{0}\right)>1$, which corresponds to an altitude $z$ of about $30 \mathrm{~km}$. However, the result for density is quite accurate for the lowest $15 \mathrm{~km}$ of the atmosphere. To remedy the errors above $15 \mathrm{~km}$, the additional terms in Equation (15) can be restored gradually above $15 \mathrm{~km}$, representing the return to true equilibrium at higher altitudes. The altitude dependent factor used to restore the full Equation (15) is given by Equation (22):

$$
f(z)=H(z-15)\left\{1-\exp \left[-(z-15)^{2} / 20^{2}\right]\right\},
$$

where $z$ is in $\mathrm{km}$ in this expression and $H(z)$ is the Heaviside function. This factor gives a gaussian onset to equilibrium and multiplies the terms $(1 / \rho)(\mathrm{d} \rho / \mathrm{d} z) m_{\text {air }} g z$ and $\mathrm{d} P / \mathrm{d} \rho /\left(r_{e}+z\right)$ in Equation (15). A numerical integration of (15) with this factor is shown in Figure 1, along with the other solutions given above. All are compared to the standard (measured) atmospheric pressure and density from [17].

Comparing the cyan and blue curves for both pressure and density in Figure 1, it is seen that the use of the full generalized equation of hydrostatic equilibrium with Equation (22) arguably gives the best fit to the measured standard atmosphere particularly for the density, and simplifies to the standard adiabatic equation below $15 \mathrm{~km}$, where strict equilibrium does not apply. It further avoids the nonsensical result given by the standard hydrostatic equation, Equation (21), above $30 \mathrm{~km}$.

\section{Solution near the Origin}

As discussed at the end of Section 1, the unconstrained equilibrium solution gives infinite density at the origin. This defies the Fermi-Dirac equation, which implies a maximum density due to fermion degeneracy pressure. It also seems counter-intuitive, at least in the absence of a gravitational singularity. 
To remedy this, a density constraint (as well as a total particle constraint) is added to the Lagrangian density, with a result given by Equation (14). It is not difficult to see that Equation (14) can be written

$$
\begin{aligned}
& \left\{\mathrm{d} \ln (\rho) / \mathrm{d} \ln (r)+1+2\left[r \lambda_{M} /\left(\rho m_{v}^{2} G\right)\right]\right\} \delta \rho(r) \\
& +\left[\left(4 \pi m_{v}^{2} G\right) r \mathrm{~d} / \mathrm{d} r(\rho / r)\right] \int_{r}^{\infty} \mathrm{d} r^{\prime} r^{\prime 2} \delta \rho\left(r^{\prime}\right) /\left[1+\rho m_{v}^{2} G /\left(r \lambda_{M}\right)\right] \\
& \times\left[1+r \lambda_{M} /\left(\rho m_{v}^{2} G\right)\right] /\left[\mathrm{d} P / \mathrm{d} \rho+m_{v} M_{e n c}(r) G / r+\lambda_{\rho}\right]=0
\end{aligned}
$$

The last term of Equation (23), comprising the last two lines, can be estimated in several ways. The simplest approach is to assume that the density variations in the integral average to zero, yielding zero for this term. However, a more careful approach is appropriate, and this can be done assuming the zeroth-order solution, Equation (13). It is further assumed $r \lambda_{M} /\left(\rho m_{v}^{2} G\right)$ is much less than 1 , to investigate behavior near the origin. Then the various factors in the last term of Equation (23) are

$$
\begin{gathered}
r \mathrm{~d} / \mathrm{d} r(\rho / r)=-2 \rho_{0} r_{0} / r^{2}, \\
1+r \lambda_{M} /\left(\rho m_{v}^{2} G\right)=1+O\left(r \lambda_{M} /\left(\rho m_{v}^{2} G\right)\right), \\
{\left[1+\rho m_{v}^{2} G /\left(r \lambda_{M}\right)\right]^{-1}=r^{2} \lambda_{M} /\left(\rho_{0} r_{0} m_{v}^{2} G\right)+o\left(r \lambda_{M} /\left(\rho m_{v}^{2} G\right)\right),} \\
\text { And } m_{v} M_{\text {enc }}(r) G / r=2 \pi m_{v}^{2} G \rho_{0} r_{0} r \equiv C_{0} r .
\end{gathered}
$$

The "big-oh" and "little-oh" notations are used in Equations (24b) and (24c). With Equations (24), the last term of Equation (23) can then be estimated as

$$
-8 \pi \lambda_{M} \int_{r}^{\infty} \mathrm{d} r^{\prime} r^{\prime 2} \delta \rho\left(r^{\prime}\right) /\left(\mathrm{d} P / \mathrm{d} \rho+C_{0} r+\lambda_{\rho}\right) .
$$

Note also that with constrained total particle number, the variations should satisfy

$$
4 \pi \int_{0}^{\infty} \mathrm{d} r^{\prime} r^{\prime 2} \delta \rho\left(r^{\prime}\right)=0,
$$

which allows Equation (25) to be re-written as

$$
+8 \pi \lambda_{M} \int_{0}^{r} \mathrm{~d} r^{\prime} r^{\prime 2} \delta \rho\left(r^{\prime}\right) /\left(\mathrm{d} P / \mathrm{d} \rho+C_{0} r+\lambda_{\rho}\right) .
$$

With bounded variations $\delta \rho\left(r^{\prime}\right)$ in 0 to $r$, this expression tends to zero as $r$ tends to zero, when the denominator is not zero (as should be the case). Hence, however the last term is estimated, the summary result for Equation (23) near the origin is

$$
\left\{\mathrm{d} \ln (\rho) / \mathrm{d} \ln (r)+1+2\left[r \lambda_{M} / \rho m_{v}^{2} G\right]\right\} \delta \rho(r)=0 .
$$

Note that this has two possible solutions for any given $r$. The first solution is obtained by the usual approach, setting the quantity inside curly brackets to zero. This gives the $r^{-1}$ solution presented in Equation (13) near the origin, 
when $r \lambda_{M} /\left(\rho m_{v}^{2} G\right)$ is much less than 1. A second solution to Equation (28) is obtained by setting the variation $\delta \rho(r)$ to zero, i.e.,

$$
\rho=\text { constant in a spherical region. }
$$

This latter solution is consistent with an incompressible medium, as occurs when the density is limited by Fermi-Dirac statistics. It is also consistent with the observed features of most celestial bodies, in which the density is approximately constant at their core, and also often in spherical shells. It is also approximately consistent with observations for dark matter in central regions that are less than a kpc in diameter [7] [9]. It implicitly assumes that the properties of the constituent materials are constant within spherical regions. It also is self-consistent with expression (27) equal to zero in such a region. With these facts in mind, the solution (29) of constant density in a region nearest the origin is a candidate solution. It should be further noted that this same result of constant density can be applied far from the origin where particles achieve their nominal cosmic background temperature and density.

Next consider the standard hydrostatic equation. One can assume a solution of the form $\rho_{0} r_{0} r^{-1}$ for the density and substitute into Equation (1) with Equation (2) and check for consistency. One finds that

$$
(1 / \rho)(\mathrm{d} P / \mathrm{d} r)=-m_{v} M_{e n c} G / r^{2} \rightarrow c_{\gamma} \gamma \rho^{\gamma-2} \mathrm{~d} \rho / \mathrm{d} r=-2 \pi m_{v}^{2} G \rho_{0} r_{0} .
$$

One notes that the left-hand side varies as $r^{-\gamma}$ whereas the right-hand side is a constant under the assumption of $\rho \sim r^{-1}$. Hence for any $\gamma$ except possibly zero the standard hydrostatic equation is not consistent with an $r^{-1}$ solution.

One can also analyze the standard equation for hydrostatic equilibrium in a gravitational field assuming constant number density $\rho_{0}$ in the vicinity of the origin of a spherical mass distribution. A constant density near the origin is self-consistent in Equation (1) as can be seen as follows. Assuming a polytropic relation $P=c_{\gamma} \rho^{\gamma}$ in Equation (1) one obtains

$$
c_{\gamma} \gamma \rho^{\gamma-2} \mathrm{~d} \rho / \mathrm{d} r=-(4 \pi / 3) m_{v}^{2} G \rho_{0} r .
$$

here $\rho_{0}$ is the sum of OM and DM mass density at the origin, normalized to the mass of the DM particles, with the assumption that OM dominates. This expression shows that the derivative of the dark or ordinary matter is zero at the origin, and gradually becomes more negative, consistent with constant density. A solution of Equation (31) for dark matter gives the following result for $\gamma>1$ :

$$
\rho(r)=\left[\rho_{01}^{\gamma-1}-\rho_{01}^{\gamma-1} C_{1} r^{2}\right]^{1 /(\gamma-1)}=\rho_{01}\left[1-C_{1} r^{2}\right]^{1 /(\gamma-1)}=\rho_{01}\left[1-\left(r / r_{a}\right)^{2}\right]^{1 /(\gamma-1)},
$$

where $\rho_{01}$ is the (equivalent) number density at the origin, and $C_{1}=(2 \pi / 3) m_{v}^{2} G \rho_{0}(\gamma-1) /\left(c_{\gamma} \gamma \rho_{01}^{\gamma-1}\right)$. Note that $C_{1}$ has units of $\cdot \mathrm{m}^{-2}$, and so one may set $C_{1} \equiv r_{a}^{-2}$, and this is used in the last equality of Equation (32). The solution is clearly approximate because for $r$ greater than $r_{a}$ the density 
can be become complex and also because the density variation of DM in Equation (32) is not included in the enclosed mass in Equation (31). However, it does show that a constant density to order of $\left(r / r_{a}\right)^{2}$ is consistent with the standard hydrostatic equation.

To summarize Section 4, it is found that setting the variations of the Lagrangian density to zero yields an alternative, constant-density solution. This solution is consistent with the observed density profile of many celestial bodies near their origin, including observationally-inferred dark matter profiles. The standard hydrostatic equation also shows solutions consistent with approximately constant density at the origin but is seen to be inconsistent with $r^{-1}$ solutions. With these considerations in mind, the constant-density solution is used in a region of radius $r_{0}$ near the origin in the following sections.

\section{Behavior of Solutions away from the Origin}

The mass-constrained Equation (14) is most relevant here. The last term in the equation is assumed negligible in this case, since the integral of any physical density variations from $r$ to infinity should tend to zero for large $r$ in the case of constrained total particle number. The result is

$$
\begin{aligned}
& {\left[1+r \lambda_{M} /\left(\rho m_{v}^{2} G\right)\right]^{-1}[(1 / \rho) \mathrm{d} \rho / \mathrm{d} r]\left[\mathrm{d} P / \mathrm{d} \rho+m_{v} M_{\text {enc }}(r) G / r+\lambda_{\rho}\right]} \\
& +\left\{2-\left[1+r \lambda_{M} / \rho m_{v}^{2} G\right]^{-1}\right\}\left[\mathrm{d} P / \mathrm{d} \rho+m_{v} M_{\text {enc }}(r) G / r+\lambda_{\rho}\right] / r=0 .
\end{aligned}
$$

Inspection shows that this equation can be simplified considerably, giving, as in Equation (28),

$$
\mathrm{d} \ln (\rho) / \mathrm{d} \ln (r)=-1-2\left[r \lambda_{M} / \rho m^{2} G\right] .
$$

This result shows that the solution is independent of the density constraint $\lambda_{\rho}$ with the neglect of the last term of Equation (14). This equation may further be put into dimensionless form by defining $\lambda_{M} /\left(m_{v}^{2} G\right) \equiv \rho_{c} / r_{c}$, where is $\rho_{c}$ the cutoff density and $r_{c}$ is the cutoff radius where the density drops due to the constraint on the total particle count. The resulting equation is

$$
\mathrm{d} \ln (\rho) / \mathrm{d} \ln (r)=-1-2\left[\left(r / r_{c}\right)\left(\rho_{c} / \rho\right)\right] .
$$

This equation may be solved numerically or perturbatively. For a perturbative treatment, assume $\left(r / r_{c}\right)\left(\rho_{c} / \rho\right)$ is much less than 1 , corresponding to radii near the origin but not at the origin. Then the zeroth order solution is given by Equation (12),

$$
\rho(r)=\rho_{0}\left(r_{0} / r\right)+O\left[\left(r / r_{c}\right)\left(\rho_{c} / \rho\right)\right]
$$

where the "big-oh" notation is used to indicate the order of the approximation. Substituting this solution back into Equation (35) gives

$$
\mathrm{d} \ln (\rho) / \mathrm{d} \ln (r)=-1-2\left[\left(r^{2} / r_{c} r_{0}\right)\left(\rho_{c} / \rho_{0}\right)\right] .
$$

This equation is readily solved to give 


$$
\begin{aligned}
\rho(r)= & \rho_{0}\left(r_{0} / r\right) \exp \left\{-\left(r^{2}-r_{0}^{2}\right)\left[\rho_{c} /\left(\rho_{0} r_{0} r_{c}\right)\right]\right\}+O\left\{\left[\left(r / r_{c}\right)\left(\rho_{c} / \rho\right)\right]^{2}\right\} \\
= & \rho_{0}\left(r_{0} / r\right)\left\{1-\left(r^{2}-r_{0}^{2}\right)\left[\rho_{c} /\left(\rho_{0} r_{0} r_{c}\right)\right]\right\}+O\left\{\left[\left(r / r_{c}\right)\left(\rho_{c} / \rho\right)\right]^{2}\right\} \\
= & \frac{\rho_{0} r_{0}}{r\left[1+\left(\frac{r^{2}}{r_{0}^{2}}-1\right)\left(\frac{\rho_{c} r_{0}}{\rho_{0} r_{c}}\right)\right]}+O\left\{\left[\left(r / r_{c}\right)\left(\rho_{c} / \rho\right)\right]^{2}\right\} \\
= & \frac{\rho_{0} r_{01}}{r\left(1+r^{2} / r_{02}^{2}\right)}+O\left\{\left[\left(r / r_{c}\right)\left(\rho_{c} / \rho\right)\right]^{2}\right\}
\end{aligned}
$$

In the last expression, $r_{01}=r_{0} /(1-\mu), r_{02}^{2}=r_{0}^{2}(1-\mu) / \mu$, and $\mu=\rho_{c} r_{0} /\left(\rho_{0} r_{c}\right)$. With $\mu$ less than 1 and $\left[\left(r / r_{c}\right)\left(\rho_{c} / \rho\right)\right]^{2}<1$, this expression is similar to the NFW result with a $1 / r$ dependence near the origin and a $1 / r^{3}$ dependence far from the origin. It is also similar to the double-power-law models and in particular the $(\alpha, \beta, \gamma)$ models [2] [3], with $(\alpha, \beta, \gamma)=(2,3,1)$. However, because the scaling factor $r_{02}$ differs from $r_{01}$, the above is formally different from such models.

To check the above results, Equation (35) may be integrated numerically from the origin for some relevant input values. In accord with Section 4 , the density is assumed constant for $r<r_{0}$. The resulting density is shown in plot (a) of Figure 2, along with approximate results from Equations (36) and (38). The dimensionless inputs for Figure 2 are $r_{0} / r_{c}=0.011$ and $\rho_{c} / \rho_{0}=0.011$. One can see that the approximation of Equation (36) is relatively good for $r$ less than about $0.5 r_{c}$ and for Equation (38) for $r$ less than about $0.7 r_{c}$. The plot on the right shows the normalized enclosed-mass curve for the numerical result in plot (a). The normalization of enclosed mass uses the results of Section 4, accounting for the region of radius $r_{0}$ of constant density $\rho_{0}$ near the origin and a $1 / r$ dependence outside this region to a radius of $0.7 r_{c}$ (at which the $1 / r$ dependence is seen to be no longer valid). The result is

$$
M_{\text {enc }, \text { tot }} \equiv 2 \pi m_{v} \rho_{0} r_{0}\left[\left(0.7 r_{c}\right)^{2}-r_{0}^{2} / 3\right] \text {. }
$$
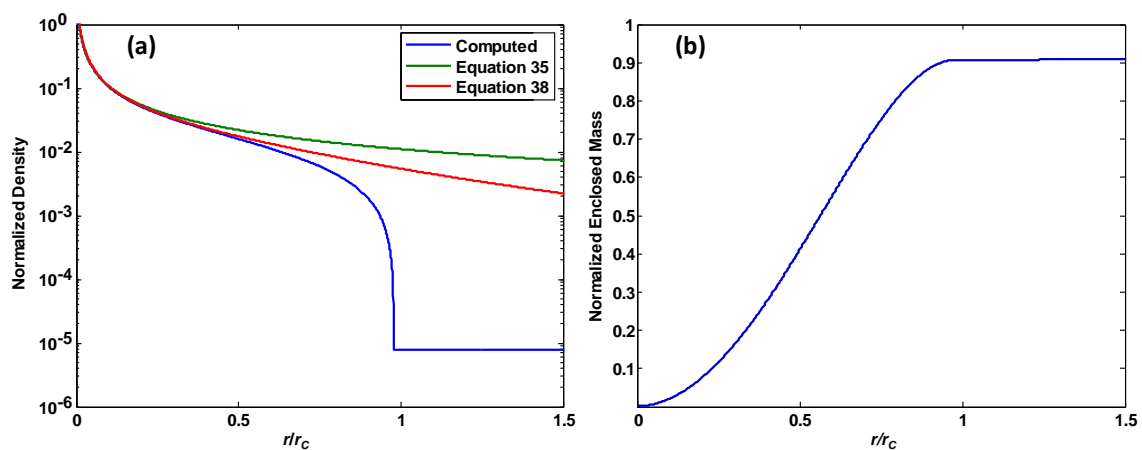

Figure 2. (a): Normalized DM density $\rho / \rho_{0}$ versus normalized radius. Blue: numerically computed from Equation (35), thresholded from below at $10^{-5} \rho_{0}$. Green: leading order result, Equation (36). Red: next leading order result, Equation (38). (b): normalized enclosed mass $M_{\text {enc }}(r) / M_{\text {enc,tot }}$ versus normalized radius for computed density profile. 
It should be noted that the results of Figure 2 are not explicitly dependent on the polytropic exponent, particle mass, or temperature. The result does depend on the latter 2 parameters implicitly through the initial density at or near the origin via Equation (4), for example. One may observe from Equation (35) that the logarithmic slope varies continuously from -1 at the origin to -3 for $r / \rho=r_{c} / \rho_{c}$. It is well known that logarithmic exponents less than -3 correspond to solutions that have convergent enclosed mass [1] [2].

In both this section and Section 4, the last term of Equation (14) is neglected with some justification. However, there is no particular justification for its neglect in regions neither near nor far from the origin. The retention of this last term could introduce perturbations into the nominal equation which are arbitrary, but which might average in some sense to zero. It should also be observed that in cases of interest, the parameter $\lambda_{M}$ is extremely small compared to the other energy densities involved, so in such cases the neglect of the last term of Equation (14) is justified. For example, with $\lambda_{M}=m_{v}^{2} G \rho_{c} / r_{c}$, with $r_{c}=92 \mathrm{kpc}$ for a halo like that of the Milky Way [19], a temperature of $2 \mathrm{~K}$ and masses of 1 $\mathrm{eV} / \mathrm{c}^{2}$ and $5 \mathrm{keV} / \mathrm{c}^{2}$, one obtains $\lambda_{M}=2.13 \times 10^{-90}$ and $1.89 \times 10^{-77} \mathrm{~J} \cdot \mathrm{m}^{-3}$, respectively, using Equation (4). These values of $\lambda_{M}$ should be compared with the pressure at the origin, which from Equations (4) and (5) is about $3.22 \times 10^{-10}$ and $1.14 \times 10^{-4} \mathrm{~J} \cdot \mathrm{m}^{-3}$ for the same two masses, respectively. Even with densities 200 times lower as occur might occur at the edge of the density profile, the value of $\lambda_{M}$ is negligible in comparison. These considerations provide some justification for neglect of the last term of Equation (14).

\section{Comparison to Einasto and De-Projected Sersic Profiles}

Taking all the above sections into consideration, one finds that $r \lambda_{M} /\left(\rho m_{v}^{2} G\right)=\left(r / r_{c}\right)\left(\rho_{c} / \rho\right)$ is always less than 1 where there is appreciable density, so the resulting solution is

$$
\rho(r)=\left\{\begin{array}{l}
\rho_{0} \text { in a spherical region about the origin of radius } r_{0}, \text { or } \\
\rho(r) \text { satisfies } \frac{\mathrm{d} \ln \rho}{\mathrm{d} \ln r}=-1-2\left(\frac{r}{r_{c}}\right)\left(\frac{\rho_{c}}{\rho}\right)
\end{array}\right.
$$

This solution has a central core region that is qualitatively consistent with the size of inner profiles of low-spectral brightness galaxies [2] [8] [9]. Further, the constant density in a core region avoids a density singularity and so is consistent with a Fermi-Dirac density distribution at finite temperature. Moreover, the finite density at the origin is consistent with all observed astronomical bodies except black holes. Lastly, the "core" solution offers a potential path to resolution to the "core-cusp" problem in dwarf galaxies.

For the region just outside the core, the logarithmic slope given by Equation (40) is -1 or less, which is consistent with most past results. A comparison of the solution to the $(\alpha, \beta, \gamma)=(2,3,1)$ model is given in the previous section. Equation (40) can also be compared to the well-known de-projected Sersic and Ei- 
nasto models [4] [5] [6]. The de-projected Sersic (dpS) density distribution for radiant matter is approximated by [2]:

$$
\rho_{\text {rad }}=\rho_{\text {rad } 0}\left(r / R_{e}\right)^{-p_{n}} \exp \left[-b_{n}\left(r / R_{e}\right)^{1 / n}\right] .
$$

The parameter $\rho_{\text {rad } 0}$ is obtained by setting the volume integral of Equation (41) equal to the measured or inferred ordinary mass of the galaxy. The variable $R_{e}$ denotes the radius which encloses $1 / 2$ the total light of the galaxy. The dpS profile is also used with some success for characterization of DM density profiles versus radius. The other two parameters in Equation (41) are given conveniently and approximately from Equations (19) and (27) of [2],

$$
\begin{gathered}
p_{n}=1.0-0.6097 / n+0.05463 / n^{2}, \text { and } \\
b_{n}=2 n-1 / 3+0.009876 / n .
\end{gathered}
$$

The parameter $n$ ranges from roughly 2 to 4 for DM haloes of galaxies and clusters, from the same reference. With these values of $n$, the leading exponent $p_{n}$ in Equation (42) is -0.7 to -0.85 , which is not too different from -1 , and [2] notes that the logarithmic slope is almost always approximated by -1 in the vicinity of the origin in the N-body simulations they investigated. The logarithmic derivative of Equation (41) is

$$
\mathrm{d} \ln \left(\rho_{d p S}\right) / \mathrm{d} \ln (r)=-p_{n}-\left(b_{n} / n\right)\left(r / R_{e}\right)^{1 / n} .
$$

Another density distribution used for characterization of DM is the Einasto distribution, which for the purposes of this paper is given by

$$
\rho_{\text {Ein }}=\rho_{0} \exp \left[-d_{n}\left(r / r_{e}\right)^{1 / n}\right] \text {, }
$$

where $r_{e}$ is the $\exp \left(-d_{n}\right)$ radius of the DM galactic halo, $n$ is typically 4 to 7 in this case, and $d_{n}$ is given approximately by Equation (24) of [2]:

$$
d_{n} \approx 3 n-1 / 3+0.0079 / n \text {, for } n>0.5 \text {. }
$$

For the Einasto distribution, the logarithmic derivative is

$$
\mathrm{d} \ln \left(\rho_{\text {Ein }}\right) / \mathrm{d} \ln (r)=-\left(d_{n} / n\right)\left(r / r_{e}\right)^{1 / n} .
$$

Inspection of Equation (44) finds a total logarithmic slope of about $-3+1 /(3 n)+0.6097 / n$ at $r=R_{e}$ for the dpS model. The leading term matches the logarithmic slope of Equation (40) at $\left(r / r_{c}\right)\left(\rho_{c} / \rho\right)=1$. Note that the onset of steeper logarithmic slopes occurs for smaller $r$ with the dpS model than for Equation (40). The primary potential disadvantage of the $\mathrm{dpS}$ model relative to Equation (40) is the infinite density at the origin (but if there is a gravitational singularity this may be an advantage).

The Einasto model does not have infinite density at the origin. The logarithmic slope of the Einasto model is also zero at the origin, matching the core solution of Equation (40). These may be viewed as positive features if the true density distribution is not infinite at the origin. However, the derivative $\mathrm{d} \rho_{\text {Ein }} / \mathrm{d} r$ is infinite at the origin for $n>1$. The logarithmic slope of the Einasto model also 
gives a value of about $-3+1 /(3 n)$ at $r=r_{e}$ using Equation (46). This is an approximate match of the logarithmic slope of Equation (40) at $\left(r / r_{c}\right)\left(\rho_{c} / \rho\right)=1$. However, as with the dpS model, the onset of steeper logarithmic slopes, slopes less than -1 but greater than -3 , occurs for smaller $r$ than for Equation (40).

This different behavior of the model solutions can be addressed with the possible solutions or extensions of Equation (14). One approach is to add angular momentum. The net effect of angular momentum would be to limit the extent of the galactic halo and would result in a more rapid decline in density than given in Equation (40). However, faster declines are found in simulations without appeal to angular momentum. As a second approach, one may consider the results of Section 3, which indicate that if the medium is not in a strict equilibrium, then solutions with faster declines occur. The outer perimeters of a large halo are not expected to be in strict equilibrium because of the process of aggregation of subhaloes [10]. Proceeding as in Section 3, one may neglect the term $(1 / \rho)(\mathrm{d} \rho / \mathrm{d} r)\left[m_{v} M_{e n c}(r) G / r\right]$ in the first line of Equation (14) and the $(\mathrm{d} P / \mathrm{d} \rho) / r$ term in the second line, as well as the last term with leading factor $\lambda_{M}$. The resulting equation is

$$
(1 / \rho) \mathrm{d} P / \mathrm{d} r=-m_{v} M_{\text {enc }}(r) G / r^{2}\left[1+2\left(r / r_{c}\right)\left(\rho_{c} / \rho\right)\right] .
$$

Consider the region $r$ which is significantly greater than $r_{0}$ but also significantly less than $r_{c}\left(\rho / \rho_{c}\right)$, to address the issue identified in the previous paragraph. Using the techniques of Equations (30) to (32), one may assume an initial $\rho_{0}\left(r_{0} / r\right)$ solution to evaluate the enclosed mass, but this time without the expectation for consistency of the resulting density profile. One obtains an equation similar to Equation (21) in this range of $r$.

$$
\begin{aligned}
\rho(r) & =\rho_{0}\left[1-r / r_{\gamma}\right]^{1 /(\gamma-1)} \\
& =\rho_{0}\left[1+r / r_{\gamma}\right]^{-1 / \gamma-1}+o\left(r / r_{\gamma}\right)+o\left[\left(r / r_{c}\right)\left(\rho_{c} / \rho\right)\right]
\end{aligned}
$$

where

$$
r_{\gamma}=c_{\gamma} \gamma /\left[2 \pi(\gamma-1) G m_{v}^{2} r_{0} \rho_{0}^{2-\gamma}\right]
$$

For related states of matter, such as neutron stars, a polytropic exponent $\gamma$ ranging from $3 / 2$ to 2 is often used [20] [21] [22]. With these choices for $\gamma$, one obtains familiar forms of solutions, $\left[1+r / r_{3 / 2}\right]^{-2}$ to $\left[1+r / r_{2}\right]^{-1}$, respectively. The former can be found as a factor in the NFW solution. The form of these solutions, along with the large values of $r_{\gamma}$ found for certain particle masses and temperatures as shown in Section 7, provides a potential explanation for the long tails observed in the Einasto and dpS model profiles. Also, in this range of $r$ one may see that $s=2 /(2-\gamma)$ can give self-consistent solutions in Equation (48) by inserting $\rho \sim 1 / r^{s}$, for $s>0$.

One may also compare the logarithmic derivative of Equation (48) to the dpS and Einasto forms. From Equation (48), the logarithmic derivative is given by

$$
\mathrm{d} \ln (\rho) / \mathrm{d} \ln (r)=-m_{v} M_{\text {enc }}(r) G / r\left[1+2\left(r / r_{c}\right)\left(\rho_{c} / \rho\right)\right] / c_{\gamma} \gamma \rho^{\gamma-1} \text {. }
$$


Substituting $\rho \sim 1 / r^{s}, s>0$, gives a right-hand side proportional to $r^{2+s(\gamma-2)}$ for $\left(r / r_{c}\right)\left(\rho_{c} / \rho\right)<0.5$. This is always negative (with the overall minus sign on the right of Equation (51)). When the right-hand side is less than -1 , the density will decline faster than the $r^{-1}$ solution. One can match the $r^{1 / n}$ dependence of the dpS and Einasto logarithmic derivatives of Equations (44) and (47) with exponent $n$ of 2 to 7 , by setting $2+s(\gamma-2)$ equal to $n^{-1}$. The requisite values of $s$ are in the range of $(13 / 7) /(2-\gamma)$ to $(3 / 2) /(2-\gamma)$, for $\gamma<2$ and in the stated range of values of $r$. Hence, the overall radial dependence of the Einasto and de-projected Sersic models can be obtained with the assumption of deviation from equilibrium, using insights from Section 3. One also observes that including the mass constraint term will invariably result in convergent mass for decreasing density with increasing radius.

\section{Lane-Emden Solutions for Non-Relativistic Particles}

It is also instructive to consider the case in which constituent particles are non-relativistic and use the standard hydrostatic relation Equation (1) and the polytropic relation Equation (2). This leads to a familiar equation for the density and provides an analytic estimate for the scale size of cosmic structures. When Equation (2) for non-relativistic fermions is inserted in the standard equation for hydrostatic equilibrium, Equation (1), one obtains

$$
(5 / 3) c_{5 / 3} \rho^{-1 / 3} \mathrm{~d} \rho / \mathrm{d} r=-m_{v} M_{e n c}(r) G / r^{2} .
$$

Multiplying Equation (52) by $r^{2}$, and then including the $\rho^{-1 / 3}$ factor in the differential, one obtains

$$
(5 / 2) r^{2} c_{5 / 3} \mathrm{~d} \rho^{2 / 3} / \mathrm{d} r=-m_{v} M_{e n c}(r) G .
$$

Dividing by $m_{v}^{2} G$ and applying the operator $r^{-2} \mathrm{~d} / \mathrm{d} r$ to both sides of Equation (53) gives

$$
\begin{aligned}
& {\left[5 c_{5 / 3} /\left(2 m_{v}^{2} G\right)\right] r^{-2} \mathrm{~d} / \mathrm{d} r\left(r^{2} \mathrm{~d} \rho^{5 / 3} / \mathrm{d} r\right)} \\
& =C_{5 / 3} \nabla^{2} \rho^{2 / 3}=-r^{-2} \mathrm{~d} / \mathrm{d} r M_{e n c}(r) / m_{v}
\end{aligned}
$$

where $C_{5 / 3}=5 c_{5 / 3} /\left(2 m_{v}^{2} G\right)$. Note that the first equality in Equation (54) assumes spherical symmetry. Account for ordinary radiant matter as part of the enclosed mass. Then

$$
M_{\text {enc }}(r)=4 \pi \int_{0}^{r} \mathrm{~d} r^{\prime} r^{\prime 2}\left[m_{v} \rho\left(r^{\prime}\right)+\rho_{m, r a d}\left(r^{\prime}\right)\right],
$$

where $\rho_{m, \text { rad }}(r)$ is the density of ordinary matter, as defined in Section 2. Also normalize the number density by its value $\rho_{0}$ at the origin. With the notatio $\rho_{1}=\rho / \rho_{0}$ the resulting dimensionless equation is

$$
\left[5 c_{5 / 3} /\left(8 \pi m_{v}^{2} G \rho_{0}^{1 / 3}\right)\right] \nabla^{2} \rho_{1}^{2 / 3}=-\rho_{1}-\rho_{m, r a d} /\left(m_{v} \rho_{0}\right) \text {. }
$$

This equation is a form of the well-known Lane-Emden equation for polytropic spheres [13] for a polytropic exponent of 5/3. The constant 


$$
L_{5 / 3}=\left[5 c_{5 / 3} /\left(8 \pi m_{v}^{2} G \rho_{0}^{1 / 3}\right)\right]^{1 / 2} \text { (non-relativistic) }
$$

has units of length and sets the scale of the decay of the density distribution. For relativistic fermions with polytropic exponent $4 / 3$ the corresponding length scale is

$$
L_{4 / 3}=\left[c_{4 / 3} /\left(\pi m_{v}^{2} G \rho_{0}^{2 / 3}\right)\right]^{1 / 2} \quad \text { (relativistic). }
$$

The parameter $L_{5 / 3}$ is shown in Table 1 as a function of particle mass for temperatures of $T=100,8$, and $2 \mathrm{~K}$ respectively at the origin. These temperatures set the density $\rho_{0}$ at the origin via Equation (4) for fermions. The temperatures larger than $2 \mathrm{~K}$ may be relevant for bound fermion states, for which the chemical potential, Fermi energy, and corresponding Fermi temperature may be much greater than ambient. Also shown is $r_{5 / 3}$ from Equation (50). The scale sizes shown for larger temperatures are much smaller because a larger temperature implies a higher density, which therefore results in a smaller scale parameter due to stronger gravitational forces from more mass. To understand the mass scaling, recall that number density scales as (particle mass) ${ }^{3 / 2}$ for non-relativistic fermions [23], so larger masses also result in larger density which further results in smaller scale sizes. Accounting for this number density scaling and the scaling of $c_{5 / 3}$ with mass, $L_{5 / 3}$ scales as $m_{v}^{-7 / 4}$ and $r_{5 / 3}$ scales as $m_{v}^{-7 / 2}$. Table 1 shows that the lower masses, $\sim 0.025 \mathrm{eV} / c^{2}$, and lower temperature, 2 to $8 \mathrm{~K}$, yield values of the scale parameter $L_{5 / 3}$ which correspond to the peak of the large-scale mass power spectrum of about $300 \mathrm{Mpc}$ [24]. Somewhat higher masses, $\sim 0.1 \mathrm{eV} / c^{2}$ and temperatures of 2 to $8 \mathrm{~K}$ correspond to scales sizes $L_{5 / 3}$ of superclusters of the order of $30 \mathrm{Mpc}$ [25] [26]. Masses of $20 \mathrm{eV} / \mathrm{c}^{2}$ or more for these temperatures give $L_{5 / 3}$ of $3 \mathrm{kpc}$ or less, corresponding to the diameter of dwarf galaxies. One may note that the temperatures and masses shown correspond to non-relativistic conditions.

Table 1. Scale parameters $L_{5 / 3}$ and $r_{5 / 3}(\mathrm{Mpc})$ versus $T$ and particle mass.

\begin{tabular}{cccccccc}
\hline$L_{5 / 3}$ & \multicolumn{7}{c}{ Mass $\left(\mathrm{eV} / \mathrm{c}^{2}\right)$} \\
\hline$T(\mathrm{~K})$ & 0.025 & 0.1 & 0.4 & 1.6 & 5.0 & 10 & 20 \\
2 & 391 & 34.7 & 3.07 & 0.27 & 0.037 & 0.011 & 0.0033 \\
8 & 275 & 24.5 & 2.17 & 0.192 & 0.026 & 0.0078 & 0.0023 \\
100 & 133 & 12.7 & 1.14 & 0.102 & 0.0139 & 0.0041 & 0.0006 \\
\hline$r_{5 / 3}$ & & & & Mass $\left(\mathrm{eV} / \mathrm{c}^{2}\right)$ & & & \\
\hline$T(\mathrm{~K})$ & 0.025 & 0.1 & 0.4 & 1.6 & 5.0 & 10 & 20 \\
2 & $3.1 \times 10^{8}$ & $2.4 \times 10^{6}$ & $1.9 \times 10^{4}$ & 147 & 2.72 & 0.240 & 0.021 \\
8 & $1.5 \times 10^{8}$ & $1.2 \times 10^{6}$ & 9390 & 73.4 & 1.36 & 0.120 & 0.011 \\
100 & $3.6 \times 10^{7}$ & $3.2 \times 10^{5}$ & 2620 & 20.7 & 0.385 & 0.033 & 0.003 \\
\hline
\end{tabular}


Table 1 shows results for $r_{5 / 3}$, assuming $r_{0}=1 \mathrm{kpc}$. The table highlights the rapid variability of this scale parameter with mass. Masses of 1.2 to $1.6 \mathrm{eV} / \mathrm{c}^{2}$ give scale sizes corresponding to the peak of the large-scale mass power spectrum, at a nominal background temperature of $2 \mathrm{~K}$, and masses of 10 to 50 $\mathrm{eV} / \mathrm{c}^{2}$ yield scale sizes that correspond to radii of large to dwarf galaxies. Note that the masses shown in this table are not consistent with recent estimates of dark-matter particle mass [27] [28], which identify masses of the order of $5 \mathrm{keV}$. This implies that some underlying assumption of this paper is not consistent with the assumptions of those results. The only key assumptions of Table 1 are (a) mechanical or energy equilibrium applies, (b) the particles are fermions, and (c) ordinary gravity applies. The value of $r_{5 / 3}$ also depends on $r_{0}$, the region in which the density is approximately constant and equal to $\rho_{0}$. This region is a corollary of the assumption of fermionic matter in the absence of a gravitational singularity.

Figure 3 shows some density profiles corresponding to Table 1. Plot (a) of Figure 3 shows results for the Lane-Emden equation, Equation (56). Plot (b) of Figure 3 shows results for the generalized hydrostatic equation, Equation (40). The assumed conditions are shown in Table 2. For the numerical integration, 4000 radial steps are used, with each step equal to $77 \mathrm{kpc}$. The seed mass at $r=0$ is set to the estimated mass of OM of a galaxy similar to the Milky Way, which is chosen to be $9 \times 10^{10}$ solar masses [19]. The chemical potential is set to zero for the results of Figure 3 because the particles are assumed to be free and non-interacting rather than bound (however, the results shown in plot (b) of Figure 3 are also consistent with bound states of matter in fluid or gaseous form). The temperature $T$ at the origin for plot (b) of Figure 3 is set to $25 \mathrm{~K}$ in order to remain above a number density floor of $10^{8} \mathrm{~m}^{-3}$ out to a radius $r_{c}$ of 300 Mpc. This choice of number density floor is based on the estimated neutrino density in standard cosmology [29]. The average temperature of the profiles on the right is about $0.15 \mathrm{~K}$ in all cases, using Equation (4) to relate density to temperature. Note also that plot (b) of Figure 3 derives the $r^{-1}$ solution. For this solution, the scale parameter $r_{\gamma}$ of Equation (50) and Table 1 is not directly relevant.

Table 2. Inputs for Figure 3.

\begin{tabular}{|c|c|c|}
\hline Input Parameter & Values & Comment \\
\hline Temperature at origin, $T$ & $2,25 \mathrm{~K}$ & $\begin{array}{l}\text { Sets density at origin using Equation (4), } \\
\qquad \mu_{v}=0\end{array}$ \\
\hline Masses of particles, $m_{v}$ & $0.025-0.3 \mathrm{eV} / c^{2}$ & \\
\hline Source mass at origin & $9 \times 10^{10}$ solar masses & Similar to Milky Way OM mass \\
\hline Inner scale $r_{0}$ & $77 \mathrm{kpc}$ & Input to Equation (40) \\
\hline $\begin{array}{c}\text { Temperature } T_{\text {out }} \text { at outer } \\
\text { radius } r_{c}\end{array}$ & $0.1 \mathrm{~K}$ & $\begin{array}{c}\text { Sets density } \rho_{c} \text { at outer radius in Equation } \\
(40) \text { using Equation (4). }\end{array}$ \\
\hline Polytropic exponent & $5 / 3$ & Input to Lane-Emden equation \\
\hline
\end{tabular}



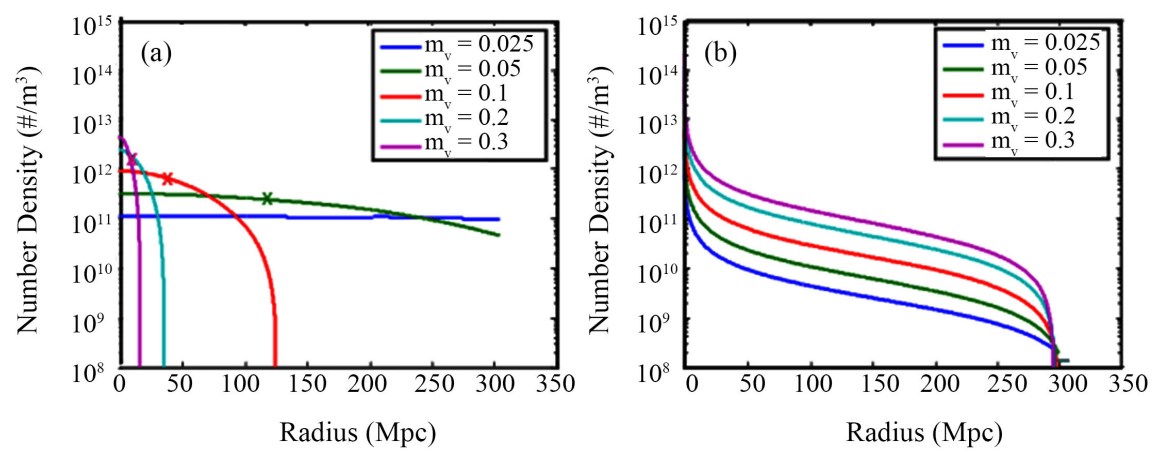

Figure 3. Particle density versus radius $(\mathrm{Mpc})$. (a) Solution to Lane-Emden equation, Equation (56), with polytropic exponent $=5 / 3$ and $T=2 \mathrm{~K}$. (b): Solution to generalized hydrostatic Equation (40) with $T=25 \mathrm{~K}, r_{c}=300 \mathrm{Mpc}$. In (a), the X's mark the corresponding large-scale structure radius $L_{5 / 3}$ from Equation (57).

In summary, this Section compares the results of the Lane-Emden equation with that of the generalized hydrostatic equation. It is seen that the former produces a different dependence of scale size on temperature and particle mass that does the generalized hydrostatic equation, and the shapes of the solutions are significantly different near the origin; the former is flat, the latter has a cusp at or near the origin. However, in both cases, a narrow range of masses and temperatures yield scale sizes that are consistent with observed galactic and large-scale structure as seen from Table 1.

\section{Summary}

In summary, Section 2 shows a derivation of a generalized hydrostatic equation in spherically symmetric geometries with a single species of matter satisfying a polytropic relation. Section 3 provides a partial validation of the generalized hydrostatic Equation (10) using the earth's atmosphere. Section 4 shows that there is a constant-density solution for the Lagrangian formulation in a spherical region about the origin (or in spherical regions about the origin), and that the $r^{-1}$ solution near the origin is not self-consistent with the standard hydrostatic equation, as expected, when the added terms of the generalized equation are not included. Section 5 explains how Equation (14) gives a convergent enclosed mass far from the origin and shows an example solution of the differential equation. Section 6 demonstrates that the solutions can match the radial behavior of accepted models of OM and DM density versus radius away from the origin. Section 7 presents solutions of the generalized equation for the density and compares it to the standard hydrostatic equation with a polytropic exponent in a gravitational potential. The overall conclusion is that a generalized equation for hydrostatic equilibrium, Equation (40), gives a cuspy solution for DM that is more consistent with observations and simulations (e.g., [1] [2]) than the standard hydrostatic equation near the origin and more consistent with finite density and lower slope in the immediate vicinity of the origin [7] [9] than the de-projected Sersic or Einasto models. Because this paper shows that the $r^{-1}$ den- 
sity profile is largely independent of the properties of the constituent material, this profile should indeed be "ubiquitous," as discussed by other authors, wherever there is strict equilibrium.

Portions of this work were presented in Paper APR19-000356 at the 2019 April Meeting of the American Physical Society.

\section{Conflicts of Interest}

The author declares no conflicts of interest regarding the publication of this paper.

\section{References}

[1] Navarro, J.F., Frenk, C.S. and White, S.D.M. (1996) The Astrophysical Journal, 462, 563-575. https://doi.org/10.1086/177173

[2] Merritt, D., Graham, A.W., Moore, B., Diemand, J. and Terzic, B. (2006) The Astronomical Journal, 132, 2685-2700. https://doi.org/10.1086/508988

[3] Hernquist, L. (1990) The Astrophysical Journal, 356, 359-364. https://doi.org/10.1086/168845

[4] Sérsic, J.L. (1963) Boletín de la Asociación Argentina de Astronomía, 6, 41-43.

[5] Prugniel, P. and Simien, F. (1997) Astronomy and Astrophysics, 321, 111-122.

[6] Einasto, J. (1965) Alma-Ata, 5, 87.

[7] Graham, A., Merritt, D., Moore, B., Diemand, J. and Terzic, B. (2006) The Astronomical Journal, 132, 2701-2710. https://doi.org/10.1086/508990

[8] de Blok, W.J.G. (2010) Advances in Astronomy, 2010, Article ID: 789293. https://doi.org/10.1155/2010/789293

[9] Oh, S.-H., Hunter, D.A., Brinks, E., et al. (2015) The Astronomical Journal, 149, 180-276. https://doi.org/10.1088/0004-6256/149/6/180

[10] Zavala, J. and Frenk, C.S. (2019) Galaxies, 7, 81-135. https://doi.org/10.3390/galaxies7040081

[11] Robertson, A., Massey, R. and Eke, V. (2017) Monthly Notices of the Royal Astronomical Society, 465, 569-587. https://doi.org/10.1093/mnras/stw2670

[12] Ringwald, A. and Wong, Y.Y.Y. (2004) Journal of Cosmology and Astrophysics, 12, 005. https://doi.org/10.1088/1475-7516/2004/12/005

[13] Lane, J.H. (1870) American Journal of Science, 2, 57-74. https://doi.org/10.2475/ajs.s2-50.148.57

[14] Graham, A.W. and Driver, S.P. (2005) Publications of the Astronomical Society of Australia, 22, 118-127. https://doi.org/10.1071/AS05001

[15] Graham, A.W. and Spitler, L. (2009) Monthly Notices of the Royal Astronomical Society, 397, 2148-2162. https://doi.org/10.1111/j.1365-2966.2009.15118.x

[16] Weinzirl, T., Jogee, S., Khochfar, S., Burkert, A. and Kormendy, J. (2009) The Astrophysical Journal, 696, 411-447. https://doi.org/10.1088/0004-637X/696/1/411

[17] White, F.M. (1986) Fluid Mechanics. McGraw-Hill, New York.

[18] ICAO (1993) Manual of the ICAO Standard Atmosphere. 3rd Edition, Montreal.

[19] Kafle, P.R., Sharma, S., Lewis, G.F. and Bland-Hawthorn, J. (2014) The Astrophysical Journal, 794, 59. https://doi.org/10.1088/0004-637X/794/1/59

[20] Ruffert, M. and Janka, H.-T. (2010) Astronomy and Astrophysics, 514, A66. 
https://doi.org/10.1051/0004-6361/200912738

[21] Cho, H.S. and Lee, C.H. (2010) Publications of the Astronomical Society of Japan, 62, 315-321. https://doi.org/10.1093/pasj/62.2.315

[22] Ozel, F. and Freire, P. (2016) Annual Reviews of Astronomy and Astrophysics, 54, 401-440. https://doi.org/10.1146/annurev-astro-081915-023322

[23] Koester, D. and Chanmugam, G. (1990) Reports on Progress in Physics, 53, 837-915. https://doi.org/10.1088/0034-4885/53/7/001

[24] Percival, W.J., Nichol, R.C., Eisenstein, D.J., Frieman, J.A., Fukugita, M., et al. (2007) The Astrophysical Journal, 657, 645-663. https://doi.org/10.1086/510615

[25] Gregory, S.A. and Thompson, L.A. (1978) The Astrophysical Journal, 222, 784-799. https://doi.org/10.1086/156198

[26] Oort, J.H. (1983) Annual Reviews Astronomy and Astrophysics, 21, 373-428. https://doi.org/10.1146/annurev.aa.21.090183.002105

[27] Iršič, V., Viel, M., Haehnelt, M.G., Bolton, J.S., Cristiani, S., et al. (2017) Physical Review D, 96, Article ID: 023522. https://doi.org/10.1103/PhysRevD.96.023522

[28] Hsueh, J.-W., Enzi, W., Vegetti, S., Auger, M.W., Fassnacht, C.D., et al. (2019) Monthly Notices of the Royal Astronomical Society, 492, 3047-3059. https://doi.org/10.1093/mnras/stz3177

[29] Perkins, D.H. (2000) Introduction to High Energy Physics. 4th Edition, Cambridge University Press, Cambridge. https://doi.org/10.1017/CBO9780511809040 